\title{
Simulation of ASDEX Upgrade Ohmic Plasmas for SOLPS Code Validation
}

\author{
A.V.Chankin, D.P.Coster, R.Dux, Ch.Fuchs, G.Haas, A.Herrmann, L.D.Horton, \\ A.Kallenbach, B.Kurzan, H.W.Müller, R.Pugno, M.Wischmeier, E.Wolfrum, \\ and the ASDEX Upgrade Team
}

Max-Planck-Institut für Plasmaphysik, Boltzmannstr. 2, 85748 Garching, Germany

\begin{abstract}
SOLPS simulation of a dedicated ASDEX Upgrade Ohmic shot for scrape-off layer and divertor modelling is described. The tendency of the code to predict colder and denser divertor plasma compared to the experiment for solutions that satisfy main boundary conditions (on the input power across the core/edge boundary, particle throughput and radiated power) and match upstream profiles is confirmed. Issues related to the role of neutral sources and sinks are discussed. In the interpretative modelling based on experimental upstream profiles, neither the neutral pressure in the divertor, nor the method of fuelling were found to strongly influence parameters at the outer target, which are primarily determined by the input power and separatrix density.
\end{abstract}




\section{Introduction}

Two-dimensional (2D) fluid codes are widely used for modelling of edge tokamak plasmas. They typically cover outer regions of the plasma core (just inside of the separatrix), scrapeoff layer (SOL) and divertor. The codes can either be used in the interpretive mode, where they match some experimentally measured parameters and profiles (the latter is usually achieved by choosing ad-hoc perpendicular anomalous transport coefficients) while reconstructing others, or in the predictive mode, where they predict plasma parameters under some (often extreme) conditions or in next generation experiments. SOLPS is one of the 2D fluid codes used for edge modelling. It is based on the B2 plasma fluid code (which includes parallel transport equations in the strongly collisional limit, but with some kinetic corrections) and a kinetic Monte Carlo code Eirene to calculate the neutral particle distribution [1-4]. This code (SOLPS version 4.2) is used extensively for predicting divertor conditions in ITER [5].

The complexity of 2D edge fluid codes requires their validation against existing well documented experiments. For SOLPS, this work was carried out using ASDEX Upgrade (AUG) plasmas with different confinement properties, and the accuracy of the code to predict parameters in the divertor was confirmed, to within a factor $2[3,4,6]$. Recently, the code validation was continued by the simulation of two AUG discharges that, out of the whole database, had the best coverage of both SOL and divertor plasmas by edge diagnostics: H-mode shot \#17151 [7] and Ohmic shot \#18737 [8]. The modelling strategy consisted of matching well-resolved upstream (far away from the divertor, along field lines) SOL profiles of electron density $\mathrm{n}_{\mathrm{e}}$, electron and ion temperatures $\mathrm{T}_{\mathrm{e}, \mathrm{i}}$, by the choice of anomalous transport coefficients, and then comparing the code results with available experimental profiles in the divertor. The full predictive power of SOLPS was mobilized, running the code in the coupled Eirene and B2.5 mode (SOLPS version 5.0), with B2.5 including main impurities and, in difference to the original version B2, classical cross-field drifts (referred to as simply 'drifts' throughout the paper). The simulations revealed a systematic tendency for the code solutions to overestimate the recycling level and $\mathrm{n}_{\mathrm{e}}$ in the divertor and to underestimate $T_{e}$. The related discrepancies in the radial electric field [9] and parallel ion flow [10] in the SOL were also established. The last two references contain discussions about possible causes of these discrepancies.

The code validation work depends critically on the quality of experimental data. The amount of data obtained should exceed the necessary minimum required for setting up the unique modelling case, thereby making the problem over-constrained, so that code results could be confronted with the experiment. Given a certain degree of uncertainty in the interpretation of experimental data and sometimes conflicting information supplied by different diagnostics 
(often caused by diagnostic malfunction), as much as possible experimental information should be collected, especially in the divertor and at the target plates, so as to eliminate any ambiguity in the treatment of experimental data. Ideally, all diagnostic signals should agree with each other (e.g., the target power should be the same when evaluated from target Langmuir probes, IR-camera and power balance calculations), and the quality of experimental profiles in the SOL should severely limit the degree of freedom in fitting code profiles to the experiment. In practice, these requirements are difficult to fulfil without careful planning of the experiment, using repeated shots in order to obtain all necessary experimental data.

The conclusion about the discrepancies between SOLPS and Ohmic plasmas at typical, medium densities, was based on the analysis of only one AUG shot \#18737. This was the best Ohmic shot out of the whole database in terms of the quality of measured upstream profiles. The validity of this conclusion could however be questioned. It is well known that fluid code solutions for the divertor depend on the density (or pressure) of neutrals in this region, which in turn depend on the geometry of the sub-divertor region, the connection between the two regions and effective pumping speed (see discussion in Section 3). In AUG, the latter is affected by the presence of material obstacles for neutrals on their path from the divertor to the pumps (see Section 3). Their influence on the effective pumping speed and neutral flux (often referred to as 'neutral pressure', however, the primary quantity measured with ASDEX pressure gauges is neutral flux) in the divertor is difficult to assess and incorporate into a $2 \mathrm{D}$ code such as Eirene. An additional complication for the comparison between SOLPS modelling of the shot \#18737 and experimental data was the lack of spectroscopic measurements at the outer divertor target due to an elevated position of the strike point, so that the divertor spectroscopic chords missed the main area of plasma interaction with the target. Hence, a direct confirmation of higher recycling in the divertor predicted by the code, based on the comparison between predicted and measured $\mathrm{H}_{\alpha}$ profiles, could not be done.

Compared to our previous edge modelling of $\mathrm{AUG}$, the present paper now includes comparisons with spectroscopic data and a more detailed modelling of the cryo-pump with Eirene. In order to collect the missing spectroscopic data, a dedicated series of Ohmic AUG discharges was carried out in the '2006 experimental campaign. Details of these experiments and SOLPS modelling focussing on high density plasmas with detachment, are described in $[11,12]$. The code benchmarking against experimental data for a medium density shot $\# 21320$, including the effect of spatial shifts (along major radius $R$ ) between upstream $n_{e}$ and $\mathrm{T}_{\mathrm{e}}$ profiles, is described in Sections 2 and 4 of the present paper. In addition, some important relations between upstream and divertor parameters, aimed at assessing the sensitivity of the code solutions to divertor design and the method of fuelling, are established in Section 3 
(results obtained in Sec. 3 give more credit to the modelling presented in Sec. 4). The summary of the work is given in Section 5 .

\section{Basic plasma parameters and setup of the SOLPS case}

The new equilibrium, with the lowered position of the outer strike point, necessary to obtain spectroscopic profiles at the outer target (SPO diagnostic) is presented in Fig. 1. In the steady-state phase of the shot \#21320, used for the simulations described in this paper, the plasma current was $1 \mathrm{MA}$, toroidal field $2 \mathrm{~T}$, line-average density across the central horizontal chord $\bar{n}_{e}=3.8 \times 10^{19} \mathrm{~m}^{-3}$. The level of density can be qualified as 'medium' for AUG Ohmic shot conditions. Choosing this density for SOLPS simulations ensures that the divertor is already in a high recycling condition, but specific modelling issues caused by the progressive detachment process can still be largely avoided (see e.g. [12]). Also, such medium density Ohmic AUG plasmas have upstream separatrix collisionalities close to that expected in

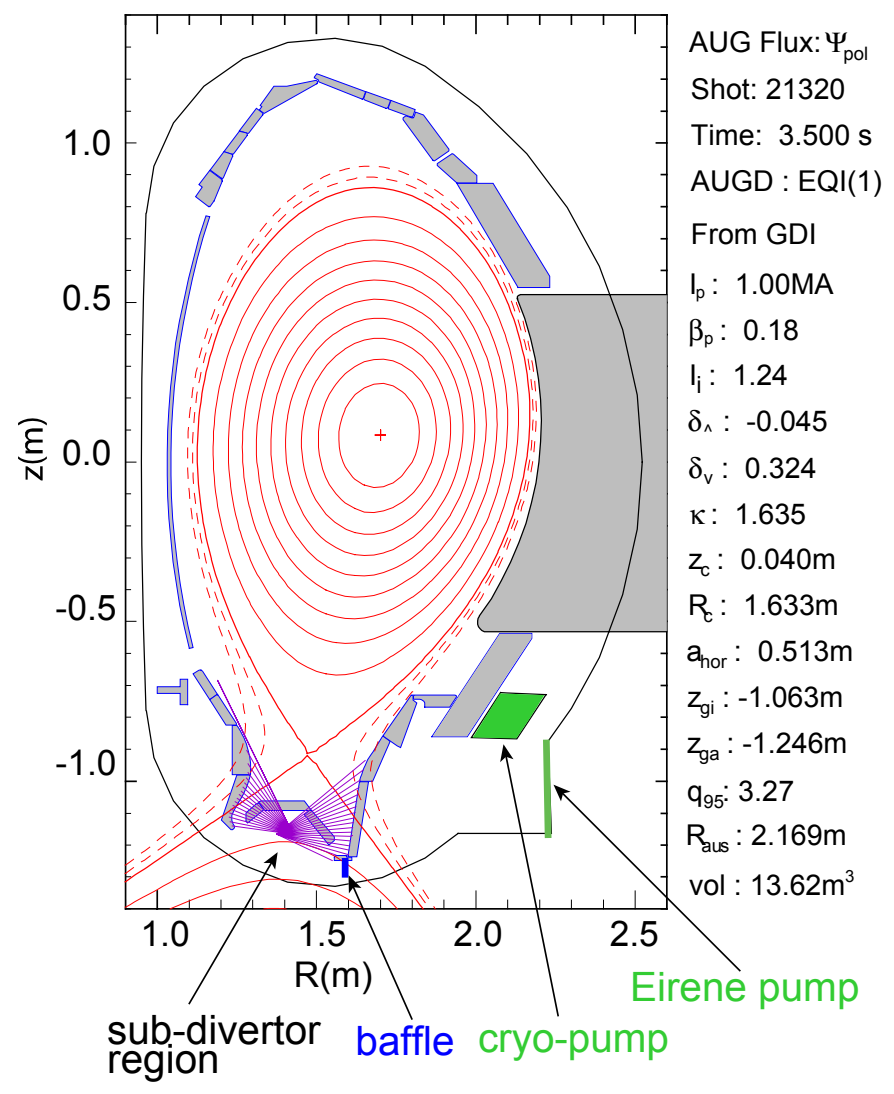

Fig. 1. Magnetic equilibrium used for Ohmic AUG shot \#21320 and details of the wall and divertor structures. Also shown are a few spectroscopic chords for measuring $H_{\alpha}$ and CIII emission profiles in the divertor. the ITER H-mode reference scenario [8], which makes their simulation relevant in light of the influence of non-local kinetic effects on the parallel electron transport regarded as one of possible explanations for the discrepancies between the $2 \mathrm{D}$ edge fluid codes and experiments $[9,10]$. As in previous simulations described in [7,8], drifts were switched on, and impurities, generated by chemical sputtering, were included in the modelling for all cases presented in Sec. 4. There were however no drifts and impurities in the cases described in Sec. 3 (see explanations below).

The $48 \times 18$ numerical grid used for SOLPS modelling is shown in Fig. 2. The input power into the computational domain from the core, shared equally between ion and electron channels, was taken as $0.8 \mathrm{MW}$, by subtracting the bulk radiation power $(0.12 \mathrm{MW})$ from the Ohmic heating power $(0.92 \mathrm{MW})$. The density was maintained by gas puffing from outside of 
the plasma at the rate of $2.4 \times 10^{21} \mathrm{~s}^{-1}(384 \mathrm{~A})$ particles per second, in terms of $\mathrm{D}$ atoms. Pumping was activated mainly by the toroidally symmetric cryo-pump $\left(120 \mathrm{~m}^{3} \mathrm{~s}^{-1}\right)$. Using readings of 3 neutral pressure gauges in the pumping region (gauges 5, 8 and 16, see Fig. 3 of [7] for details; the gauges are at different toroidal positions, but show very similar readings), the total pump-out of neutrals was estimated as $2.15 \times 10^{21} \mathrm{~s}^{-1}$ (345 A). In the SOLPS simulations, the gas puff was fixed at the experimental level in all cases, and neutrals were pumped out. For Eirene neutral particles, vacuum (no plasma) was assumed outside of the SOLPS grid. To simulate the effect of the cryo-pump, the toroidally symmetric 'Eirene pump' with the total pumping surface of $2.45 \mathrm{~m}^{2}$ (shown in Fig. 1, alongside with the cryo-pump) was introduced in Eirene. The albedo of this pump, 0.15, was chosen so as to match the productivity of the cryo-pump. The exact geometry used in the Eirene Monte-Carlo calculations for neutrals can be found in Fig. 3 of Ref. [7].

The separatrix density at the outer midplane $\mathrm{n}_{\text {sep }}$ was used to control density in the SOL and divertor. Its

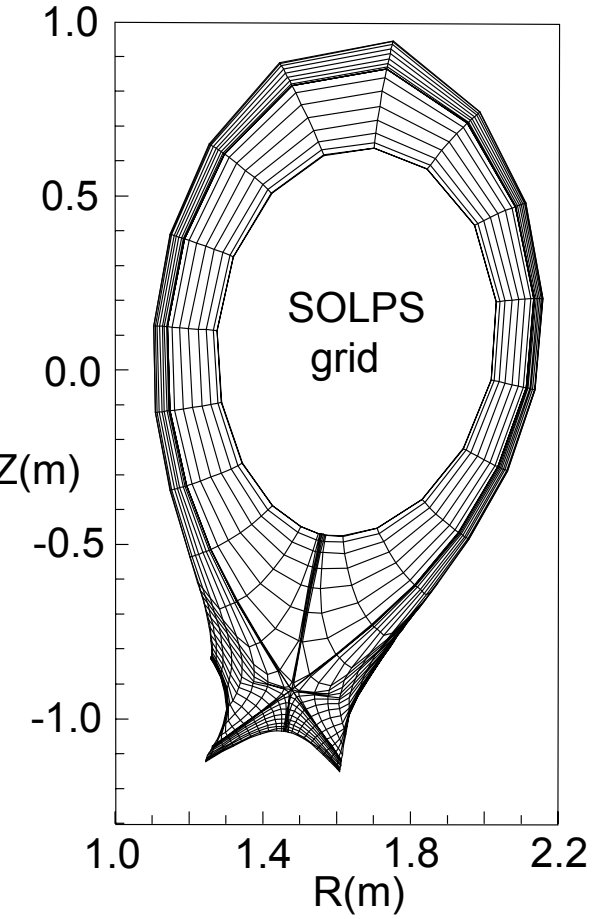

Fig. 2. $48 \times 18$ numerical grid used for SOLPS modelling. level was maintained by the (usually small) fuelling from the core via a feedback control by varying the density at the innermost core ring. A $100 \%$ recycling at the wall and divertor targets was assumed in the modelling. The radiated power within the computational domain $\mathrm{P}_{\mathrm{rad}}$ was estimated as $0.43 \mathrm{MW}$ from bolometer measurements. In the simulations, it could be achieved by varying the recycling level in the divertor by changing $\mathrm{n}_{\text {sep }}$ (radiation on the main working gas, deuterium) and carbon chemical sputtering from all plasma facing surfaces. Although nearly all plasma exposed surfaces, except for target tiles, were covered with $\mathrm{W}$ in these experiments, no strong change in $\mathrm{C}$ influxes and $\mathrm{C}$ content could be observed, the wall was covered with thin hydro-carbon films and carbon was the main radiating impurity [13]. In B2.5, multiple charge states of carbon were followed, in addition to deuterium as the main working gas. Neutrals were modelled by the Eirene version, dated 1999, which included elastic $\mathrm{D}_{2}-\mathrm{D}^{+}$collisions and a crude model to account for vibrational states of $\mathrm{D}_{2}$. Neutralneutral collisions were not important for divertor densities in the modelled AUG Ohmic plasmas and were not included in Eirene.

SOLPS fits to the experimental upstream profiles in the SOL were obtained by the choice of ad-hoc anomalous perpendicular transport coefficients $D_{\perp}, \chi_{\mathrm{e}}$ and $\chi_{\mathrm{i}}$. High quality upstream 
$\mathrm{n}_{\mathrm{e}}$ profiles were obtained from Li beam diagnostic, and high quality $\mathrm{T}_{\mathrm{e}}$ profiles - from the ECE radiometer. The $\mathrm{Li}$ beam also measures $\mathrm{T}_{\mathrm{i}}$ profiles, but with a much poorer quality in Ohmic AUG plasmas. The spatial link between $n_{e}$ and $T_{e}$ profiles can be established using YAG Thomson laser scattering data that provides both $n_{e}$ and $T_{e}$ profiles. Due to a restriction on the total number of available (vertical) laser beams, two shots are required to obtained the profiles across the whole plasma, with the beam lines covering separately core and edge regions of the plasma. The YAG diagnostic, however, does not always deliver necessary quality of the profiles at the edge so that they could be superimposed onto Li beam and ECE radiometer profiles, with the aim of establishing geometrical shifts between these two diagnostics. This was the case for the series of shots for edge modelling described in $[11,12]$, including the shot \#21320 analysed in this paper (see more on this in Sec. 4) The relative position of the $n_{e}$ profile measured by the Li beam with respect to the $T_{e}$ profiles measured by the ECE radiometer could only be determined with the $8 \mathrm{~mm}$ precision. Possible shifts between the profiles by such a small distance was however critical for SOLPS solutions in the divertor due to sharp density and temperature gradients around the separatrix position. As a result, a family of SOLPS solutions could be obtained, depending on the value of a small shift between $n_{e}$ and $T_{e}$ profiles, and additional experimental information, e.g. power balance considerations and readings of neutral pressure gauges surrounding the plasma, had to be used to select the most realistic solution. The fitting of experimental upstream $n_{e}$ and $T_{e}$ profiles in the SOLPS code runs will be presented in Section 4.

\section{Influence of neutral pumping and sources on SOL and divertor solutions}

One of the goals of the modelling described here was a more realistic description of neutral penetration from the divertor to the pumping region and their subsequent pump-out. In earlier SOLPS modelling, the neutral pressure drop between the sub-divertor region (see Fig. 1) and the pumping region was $\approx 2: 1$. This was in contrast with the neutral pressure gauges data showing a much more pronounced drop, in the range 6-7:1. The discrepancy was attributed to blockages of the path between the two regions, mainly by cables. To account for this, an artificial 'baffle' was introduced in Eirene (see Fig. 1). Thus, instead of adjusting the pumping efficiency in the modelling, as was done in earlier modelling, the baffle width was varied until the agreement with the experimental neutral pressure drop was reached, while the efficiency of the Eirene pump was fixed at the experimental level. In SOLPS runs, the influence of drifts and impurities on the neutral flux ratio was found to be insignificant. They can however influence divertor and target parameters, and drifts in particular are known to affect asymmetries between the targets. The presence of both was however found to be not important for relative changes of divertor and target parameters caused by the variation of the baffle width. At the same time, their inclusion in the SOLPS modelling significantly increased the CPU consumption. For these reasons, drifts and impurities were not included in 
the baffle width scan presented in this section. They were also deemed not important for the comparison between the two different plasma fuelling methods described later in this section. This comparison was done using a finer mesh which by itself greatly increased the CPU consumption.

The results of the SOLPS baffle width scan, with fixed separatrix density $\mathrm{n}_{\text {sep }}$ and transport coefficients, are presented in Figs. 3-4. SOLPS cases fitted very well experimental $n_{e}$ and $T_{e}$ profiles, where the $T_{e}$ profile was exactly the same as shown in the next section, but the $n_{e}$ profile was slightly different: the baffle width scan was carried out before the latest refinement of the $\mathrm{Li}$ beam $\mathrm{n}_{\mathrm{e}}$ profile data using the Bayesian analysis (see next section) was done. The value of $n_{\text {sep }}$ was chosen to be $1.4 \times 10^{19} \mathrm{~m}^{-3}$ in these runs, which is higher than the $1.3 \times 10^{19} \mathrm{~m}^{-3}$ value used for the previous simulation of the standard AUG Ohmic case described in [8] (the line average density and input power across the core/edge boundary of the computational domain were higher than in the previous shot: $3.8 \times 10^{19}$ vs. $3.65 \times 10^{19} \mathrm{~m}^{-3}$ and 0.8 vs. $0.52 \mathrm{MW}$, respectively. The difference in the input powers was mainly due to the difference in plasma currents: 1.0 vs. $0.8 \mathrm{MA}$ ).

Fig. 3a shows the ratio between sub-divertor and pumping region neutral fluxes calculated by Eirene in the baffle width scan. The ratio increases from 1.65 to 50.7 , as the baffle width is increased from zero (no baffle) to $98.125 \%$, in terms of the percentage of the blockage of the gap between the two regions created by the lower outer target tile and the wall (see Fig. 1).
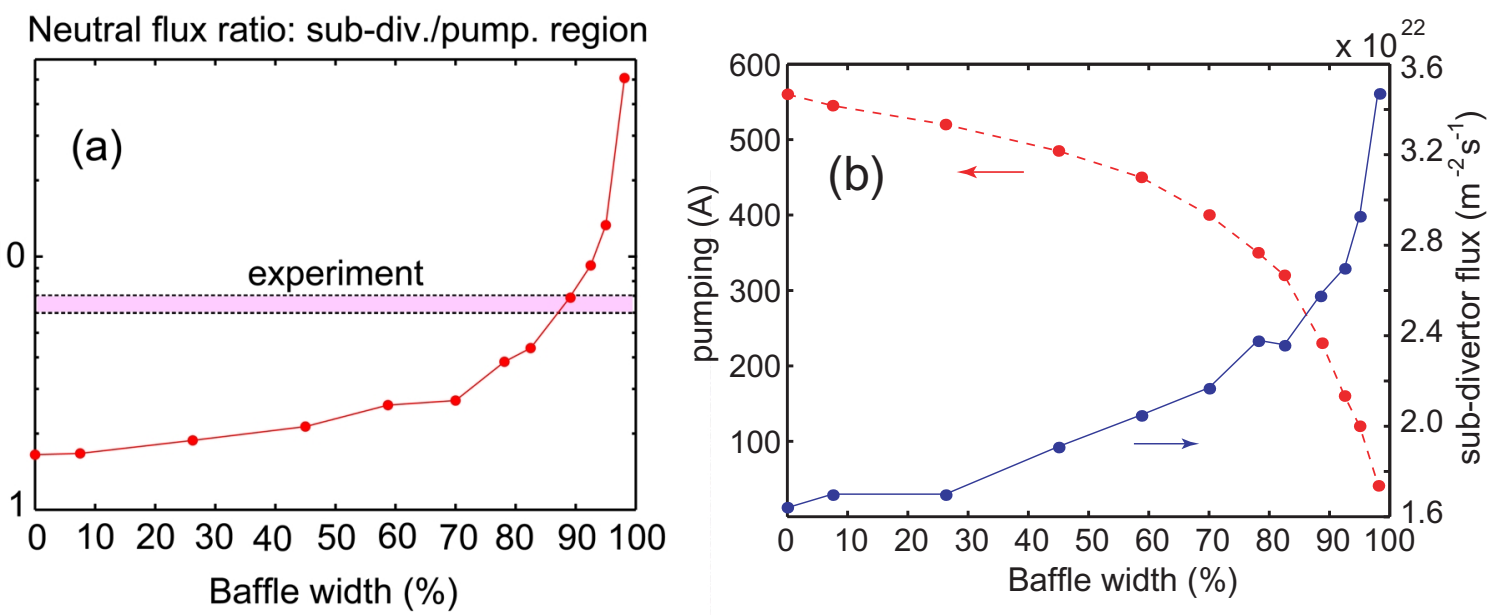

Fig. 3. Simulated neutral flux ratio between sub-divertor and pumping regions (a), pumping by the Eirene pump and sub-divertor neutral flux (b), obtained by Eirene in the baffle width scan. The experimental ratio (6 to 7) is also shown (a). The position of the baffle is indicated in Fig. 1. The $100 \%$ baffle width corresponds to the complete blockage of the path between the two regions.

The experimentally measured ratio of 6 to 7 is also indicated by a horizontal bar. From these results one concludes that nearly $90 \%$ of the gap should be closed in order to obtain experimental values of the neutral flux ratio. The baffle width was fixed at this value in the 
SOLPS cases presented in Section 4. As the baffle width is increased, the neutral pumping by the Eirene pump is reduced from 560 to $41 \mathrm{~A}$, for the baffle widths quoted earlier, while the sub-divertor neutral flux is increased by more than factor 2, see Fig. $3 b$.

Despite a substantial increase in the sub-divertor neutral flux in the baffle width scan described above, neither the upstream separatrix $T_{e}$, nor the downstream/divertor $T_{e}$, were strongly affected in this series of SOLPS runs with fixed $n_{\text {sep. }}$. This is illustrated in Fig. $4 a$, where only the peak $\mathrm{T}_{\mathrm{e}}$ at the inner target showed a noticeable reduction, from 5.84 to $3.7 \mathrm{eV}$. Other target parameters seem almost unaffected by the neutral flux. This can be seen in Fig. $4 \mathrm{~b}$, where integrated ion fluxes along each target, converted to ion saturation currents for
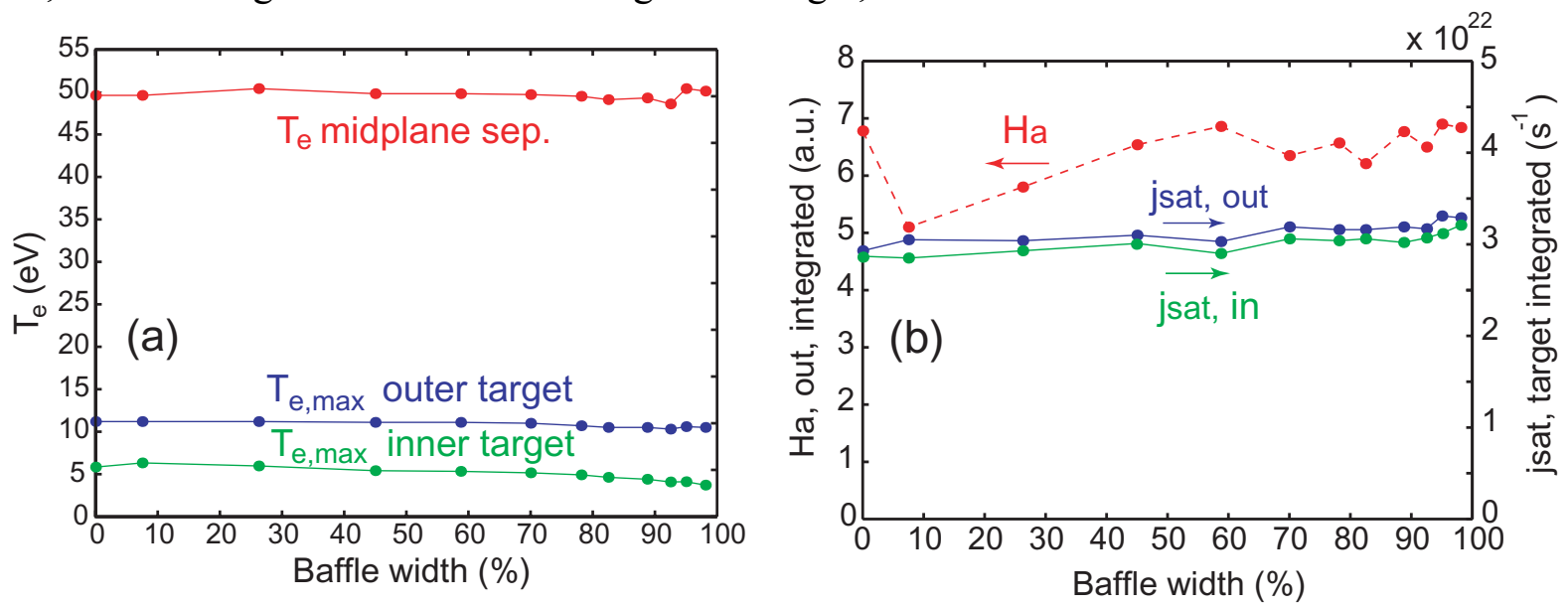

Fig. 4. Simulated separatrix $T_{e}$ at the outer midplane, peak inner and outer target $T_{e},(a)$, outer and inner integral target ion saturation currents, and the outer target integral $H_{\alpha}$ radiation (b), in the baffle width scan. See details in the caption of Fig. 3 and text.

comparison with probe measurements, show very small variation. The same applies to the simulated $\mathrm{H}_{\alpha}$ radiation integrated over the spectroscopic chords viewing the outer target, which shows little variation except for an unexplained drop at $7.5 \%$ baffle width. (For the inner target $\mathrm{H}_{\alpha}$ radiation, the simulated emission is not confined to the chords viewing the target, but extends further up, in the direction to the X-point, and this quantity is therefore less meaningful for the characterisation of inner target parameters).

Weak dependence of the outer target parameters on the sub-divertor neutral flux established in the baffle width scan may seem to be in conflict with the well known strong influence of the neutral pressure on target parameters. For example, in the B2-Eirene modelling of the ITER divertor described in [14] the dependence $p^{-0.85}$ of the power flux to the outer target on the neutral pressure $p$ was established. There is, however, no direct contradiction here, since results of such studies depend on the selection of fixed and varied parameters, and it is well known that all parameters of the edge plasma are interconnected. In particular, in the scans described in [14] the separatrix density was varied by gas puffing rather than being directly 
controlled as in the baffle width scan here, and the dependence $p^{0.36}$ was obtained for $\mathrm{n}_{\text {sep. }}$ As pointed out in [14], $\mathrm{n}_{\mathrm{sep}}$ is not the parameter that can be directly controlled in the experiment, rather, it is determined by the gas throughput and the effective pumping speed. The latter is affected by the divertor geometry and even by the strike point position on the target. For the purposes of the interpretive simulation described in this paper, however, it is the $\mathrm{n}_{\text {sep }}$, together with the input power into the computational domain, which makes the largest impact on the target parameters. It can be determined in the simulations provided upstream radial profiles of $\mathrm{n}_{\mathrm{e}}, \mathrm{T}_{\mathrm{e}}$ and $\mathrm{T}_{\mathrm{i}}$ were measured and the target power was established, either from direct measurements or from power balance calculations (see next section).
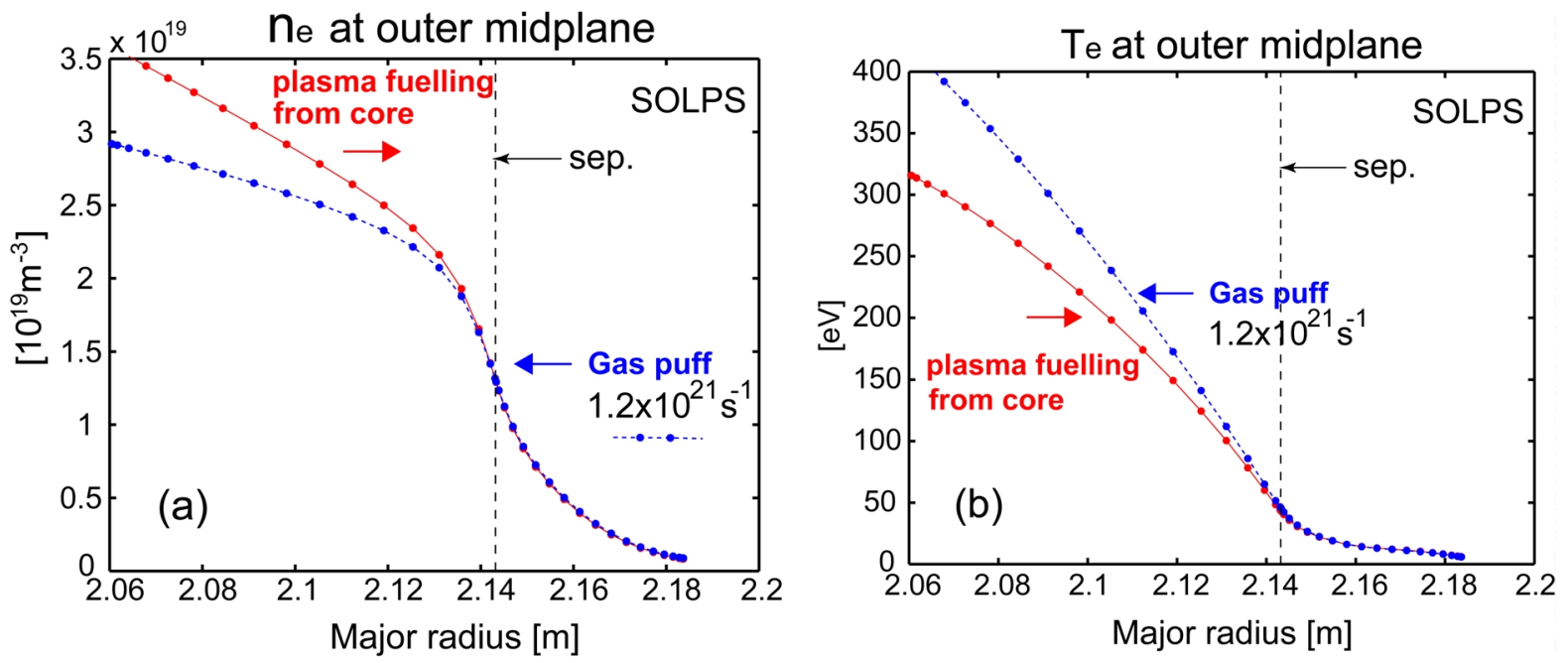

Fig. 5. Simulated electron density (a) and temperature (b) profiles at the outer midplane for the standard AUG Ohmic shot earlier simulated in [8]. Two cases are presented, for the external neutral gas puffing and plasma core fuelling methods.

Further evidence for the key role played by the separatrix density in determining target parameters was obtained in the comparison of the two SOLPS cases with different fuelling methods. The cases were a continuation of earlier simulations of the AUG standard Ohmic shot described in [8], but were run on the finer mesh $96 \times 36$. One case was run with the gas puffing of $1.2 \times 10^{21} \mathrm{~s}^{-1}$, consistent with the experiment, and the other - with the plasma fuelling from the core at a fixed $n_{\text {sep }}$ (essentially, feedback

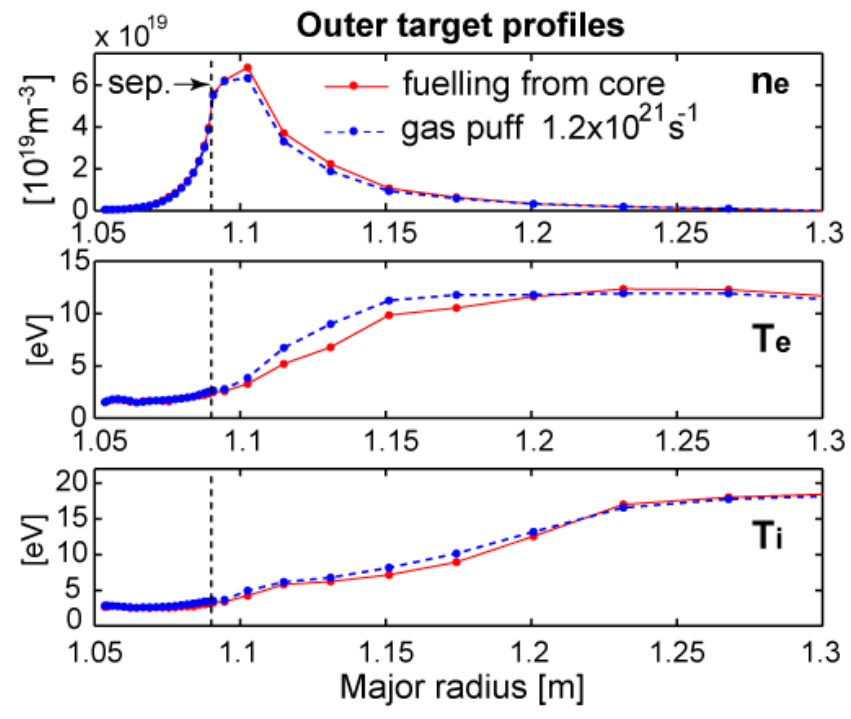

Fig. 6. Simulated outer target profiles of $n_{e}, T_{e}$ and $T_{i}$ for the two different fuelling methods. See details in the caption of Fig. 5 and text. Cases are the same as shown in Fig. 5. 
controlled as explained in the previous section) obtained in the first case. Both cases were without drifts and impurities. The results are presented in Figs. 5-6. Figs. 5a,b show upstream $\mathrm{n}_{\mathrm{e}}$ and $\mathrm{T}_{\mathrm{e}}$ profiles obtained at the outer midplane position. The profiles differ in the core, but look almost identical in the SOL. Fig. 6 shows outer target profiles of $n_{e}, T_{e}$ and $T_{i}$. Again, very little difference in the profiles can be seen, indicating a relatively minor impact of the method of fuelling on SOL and divertor parameters.

\section{SOLPS upstream profile fits and simulations of divertor conditions}

Experimental upstream (near the outer midplane position) profiles of $n_{e}$ and $T_{e}$ are presented in Figs. 7 and 11 (but note different shifts between $\mathrm{n}_{\mathrm{e}}$ and $\mathrm{T}_{\mathrm{e}}$ profiles on these figures), alongside with modelling fits to these profiles and perpendicular transport coefficients used to obtain the fits. The baffle width was fixed at $89 \%$ (see previous section) for all cases described in this section. The SOLPS $n_{e}$ profile for the case shown in Fig. 7, with the density falling towards the plasma centre, is the result of a non-physical model that allows the edge plasma to flow into the core (i.e., an inward particle flux that does not return) in order to obtain the desired separatrix $\mathrm{n}_{\mathrm{e}}\left(\mathrm{n}_{\text {sep }}\right)$ for a given (experimental) gas puff. The low $n_{\text {sep }}$ chosen for this case (in order to better match target profiles) allows too much ionisation of puffed neutrals inside the separatrix. For the given $\mathrm{n}_{\mathrm{sep}}$, pumping by the cryo-pump is insufficient to remove all these ionised neutrals, resulting in plasma "leakage" into the core via an inverted density gradient. The code $n_{e}$ profile far inside of the separatrix can therefore be ignored. For the SOL and divertor physics, it is important that the experimental separatrix $n_{e}$, its spatial derivative (along major radius) and the whole profile is the SOL are well matched by the code.

As was explained in Section 2, geometrical

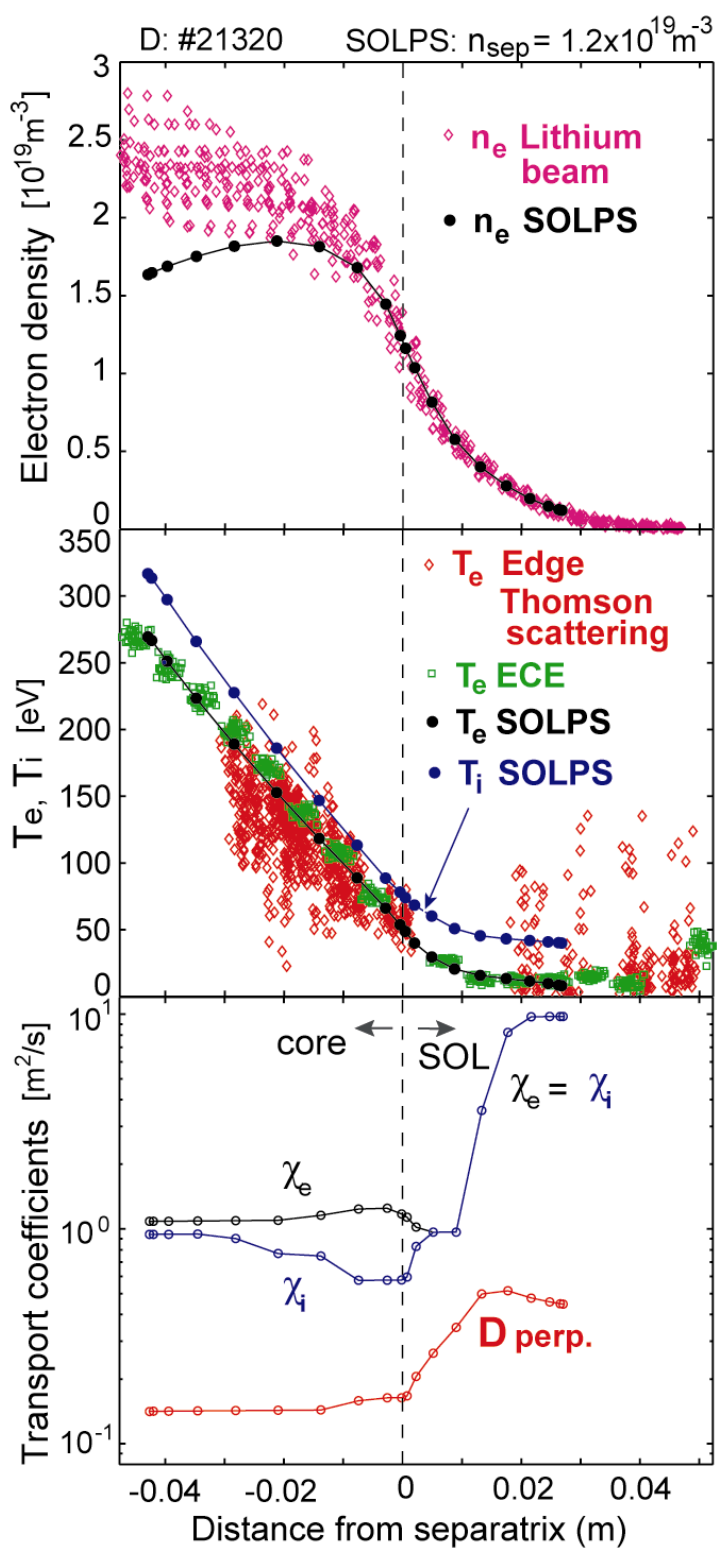

Fig. 7. Experimental and code simulated profiles of $n_{e}, T_{e}$, simulated $T_{i}$ profile, and perpendicular transport coefficients used to obtain the fits, for the case with $n_{\text {sep }}=1.2 \times 10^{19} \mathrm{~m}^{-3}$ (see text for details). 
shifts between ECE and Li beam diagnostics introduce ambiguity in the edge modelling, and, depending on the adopted shift between these profiles, a whole family of SOLPS solutions can be obtained. The $\mathrm{T}_{\mathrm{e}}$ profile measured by Thomson scattering is affected by systematic errors (related to calibration) and plasma fluctuations. The statistical error is relatively small, below $15 \%$, for plasma densities above $5 \times 10^{18} \mathrm{~m}^{-3}$, but is greatly increased at low densities, contributing to a large scatter of points in the SOL. The Thomson scattering $\mathrm{n}_{\mathrm{e}}$ profile was also affected by these errors. In addition, there was an uncertainty in the absolute value of the power of the laser beam, making these profiles unusable. They are not shown in Figs. 7 and 11. Large scatter of points on the Li beam $n_{e}$ profile inside of the separatrix is attributed to both plasma fluctuations and poor statistics due to the attenuation of the beam.

The two solutions analysed below almost completely cover the range of possible shifts between $n_{e}$ and $T_{e}$ profiles The first solution is presented in Fig. 7. It has a low separatrix electron density $\mathrm{n}_{\text {sep }}=1.2 \times 10^{19} \mathrm{~m}^{-3}$. The validity of this solution, as well as of the other (opposite, in terms of the allowable shifts between the two profiles) solution, resulting in a high density $\mathrm{n}_{\text {sep }}=1.6 \times 10^{19} \mathrm{~m}^{-3}$ (see Fig. 11), will be discussed later in this section, by comparing other available experimental data with the modelling results. The $\mathrm{n}_{\mathrm{e}}$ profile was obtained by using Bayesian probabilistic analysis of Li beam data (see [15] for details on this analysis). The $T_{i}$ profile obtained by the same diagnostic showed large scatter. The data however were not in conflict with the assumption of $T_{i} \approx T_{e}$ in the core, which in turn was in line with the results of the earlier simulated AUG standard Ohmic shot described in [8], where this approximate equality was established in the experiment due to a much smaller scatter of the $T_{i}$ data. Therefore, in the present modelling the $\chi_{i}$ profile was specified so as to achieve $T_{i} \approx T_{e}$ in the core. In the SOL, $\chi_{i}=\chi_{\mathrm{e}}$ was assumed, similar to the previous modelling [8].

Once the choice of the value of the shift between the $n_{e}$ and $T_{e}$ profiles is made, for the profiles well fitted by a SOLPS solution (as in Fig. 7, in the SOL) there exists only one possible position of the separatrix and only one value of $\mathrm{n}_{\text {sep }}$. This is due to the requirement that the simulated power to the target, conducted and convected by the plasma in the SOL, should match the experimental value. Measured and simulated powers to the outer target are presented in Fig. 8. The

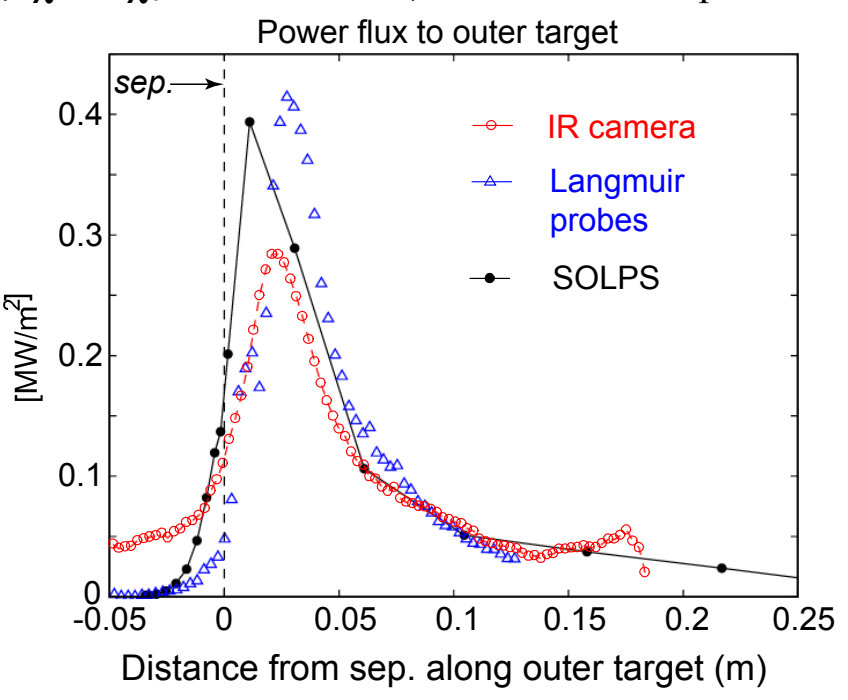

Fig. 8. Measured and simulated power flux profiles to the outer divertor target for the case with $n_{\text {sep }}=1.2 \times 10^{19} \mathrm{~m}^{-3}$. 
simulated power is similar to that estimated from the target Langmuir probe measurements using the relation $7 c_{s} n_{e} T_{e}$ for the parallel power flux, but slightly exceeds the power from the IR camera. $72 \%$ of the total simulated target power arrived at the outer target, which is typical for drift cases in medium density Ohmic AUG plasmas. The radiated power within the computational domain $\mathrm{P}_{\text {rad }}$ was $0.425 \mathrm{MW}$, very close to the experimental value. The match was achieved by the choice of the chemical sputtering coefficient of $1.15 \%$. The radiation was nearly equally split between deuterium and carbon impurities.

Measured and simulated outer target $n_{e}$ and $T_{e}$ profiles (plus the simulated $T_{i}$ profile) for the above case are shown in Fig. 9. Fairly good agreement is achieved between the measured and simulated $T_{e}$ profiles. This agreement is not coincidental, in fact, $\mathrm{n}_{\text {sep }}$ in this series of runs was reduced (by way of shifting measured upstream $n_{e}$ and $T_{e}$ profiles) until the good agreement was achieved. As was pointed out in the Introduction, SOLPS tends to predict denser and colder plasma at the outer target than in the experiment, so the goal here was to find the value of $n_{\text {sep }}$ at which the agreement between the target $T_{e}$ profiles could nevertheless be obtained, and then to check whether there was a consistency among other data. The peak simulated target $n_{e}$ is substantially higher than the measured value. This, however, is not inconsistent with the good match obtained for the outer target powers (Fig. 8), since the
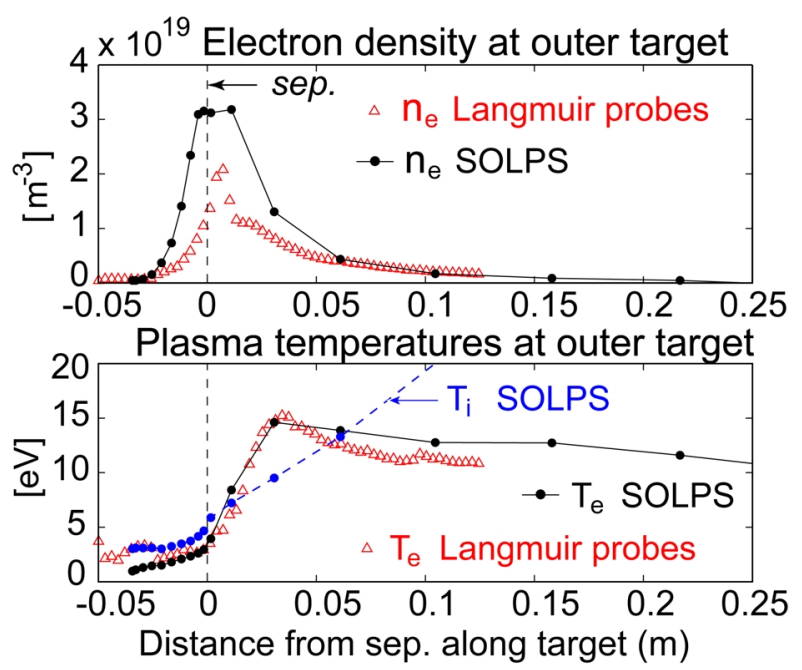

Fig. 9. Measured and simulated outer target profiles of $n_{e}$ and $T_{e}$, simulated $T_{i}$ profile, at the outer divertor target for the case with $n_{\text {sep }}=1.2 \times 10^{19} \mathrm{~m}^{-3}$.

difference in $n_{e}$ is much smaller for the positions where $T_{e}$ is the highest. At the same time, the code predicted $T_{i}$ (included in the SOLPS simulation of the target power flux via the ion channel) is smaller than $T_{e}$ at the peak of the heat flux, and $T_{e}$ was used in the formula for the power flux calculated from the Langmuir probe data. At the inner target, some probes didn't function, while others showed $\mathrm{T}_{\mathrm{e}} \approx 2 \mathrm{eV}$, being in the range of electron temperatures where the probe data are known to be unreliable. The simulated $\mathrm{T}_{\mathrm{e}}$ near the strike point position on the inner target is within $1-2 \mathrm{eV}$. In SOLPS solutions, the inner target tends to stay attached as the density is increased, despite experimental signatures of an ongoing detachment. Simulating plasma parameters at the inner target is a much more challenging task compared to the outer target, owing to a much lower power flux to this target, which greatly increases sensitivity of the code solutions to uncertainties in the input parameters of the code. In addition, there are some specific issues related to the detachment process that may explain the 
tendency of the inner target to stay attached in the code solutions. They are discussed in $[11,12]$.

$\mathrm{H}_{\alpha}$ and CIII emission profiles across the targets measured by the divertor spectroscopic array (see observation chords in Fig. 1) and simulated by SOLPS are shown in Fig. 10. At the inner target, the plasma is already in advanced stages of detachment, as discussed above. This is manifested by high $\mathrm{H}_{\alpha}$ radiation stretching far into the SOL region, in contrast to the code solution which is still mostly attached (see the discussion just above), with the peak $\mathrm{n}_{\mathrm{e}}$ of $6 \times 10^{19} \mathrm{~m}^{-3}$. Both measured and simulated CIII emissions are low at this target, owing to low $\mathrm{T}_{\mathrm{e}}$. At the outer target, the simulated $\mathrm{H}_{\alpha}$ emission is below the experimental values, but the discrepancy is not large. The simulated CIII emission, which is controlled by the choice of the chemical sputtering coefficient (with physical sputtering playing a minor role) so as to match the measured total radiated power at the edge, is below the experimental by factor 2 .
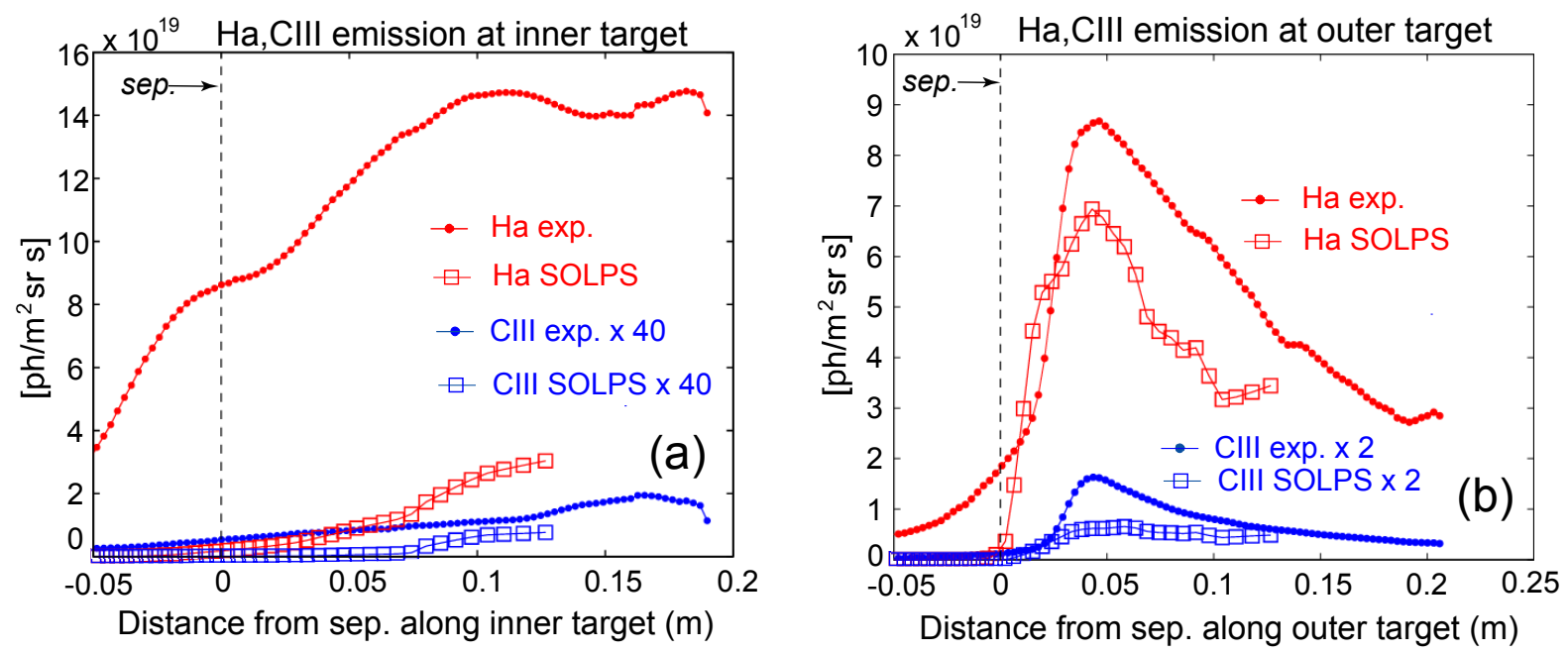

Fig. 10. Measured and simulated $H_{\alpha}$ and CIII emission profiles across inner (a) and outer (b) targets for the case with $n_{\text {sep }}=1.2 \times 10^{19} \mathrm{~m}^{-3}$. Negative distances at the inner target correspond to the private region. The position of divertor spectroscopic chords is indicated in Fig. 1.

Overall, outer target profiles of the low density SOLPS solution with $\mathrm{n}_{\mathrm{sep}}=1.2 \times 10^{19} \mathrm{~m}^{-3}$ may be regarded as being in a reasonably good agreement with the experiment, within factor 2 . A similar discrepancy exists between simulated and measured pumping rates. The SOLPS simulated pumping rate for this case is $174 \mathrm{~A}$, much below the experimental value of $\approx 345 \mathrm{~A}$ which was estimated using the pump productivity and data from neutral pressure gauges located in the pumping region (see Section 2). The gas puff rate in this discharge was $\approx 384$ A (see Section 2). The levels of experimental gas puff rate and neutral pumping implied a relatively high neutral throughput, inconsistent with the choice of the low $n_{\text {sep }}=1.2 \times 10^{19} \mathrm{~m}^{-3}$ in the SOLPS run. This is evidenced by the shape of the simulated upstream $n_{e}$ profile in Fig. 7 , with the inverse density gradient at the inner core boundary. Maintaining an artificially low 
$\mathrm{n}_{\text {sep }}$ by the feedback control via the core flux required a rather large plasma leakage into the core, $5.6 \times 10^{20} \mathrm{~s}^{-1}$, which, together with neutral leakage into the core, $6.7 \times 10^{20} \mathrm{~s}^{-1}$, made up $\approx$ $33 \%$ of the plasma flux through the separatrix into the SOL. Factor of 2 discrepancies between code simulated and measured pumping rates are not uncommon in edge plasma modelling. For example, in [16] an initial discrepancy of factor 10 in simulating Alcator CMod divertor with OSM-Eirene modelling could be reduced to factor 2 after implementation of a more realistic neutral model in Eirene. The remaining factor 2 discrepancy was therefore regarded as a relative success, and it was hoped that further refinement of the plasma solutions together with a continuing review of the experimental data would eliminate this discrepancy. The situation is however different with the simulation described here. Not only the simulated pumping rate is lower than the measured one, in spite of higher predicted target density, but the low simulated pumping rate resulted in a non-physical upstream $n_{e}$ profile, as disscussed above. At the same time, the potential for a downward revision of the experimental pumping rate is rather limited in AUG: the pumping speed of the torus pumping system has been absolutely calibrated, and the accuracy of the gas balance within $10 \%$ can be established using distributed pressure gauges (see e.g. [17]).

In order to match the experimental pumping rate, the separatrix density had to be increased up to $\mathrm{n}_{\mathrm{sep}}=1.6 \times 10^{19} \mathrm{~m}^{-3}$. This was achieved by shifting the experimental $n_{e}$ profile towards larger major radii with respect to the $T_{e}$ profile by $6.5 \mathrm{~mm}$, and then re-fitting the experimental profiles with the SOLPS solution by specifying different transport coefficients, see Fig. 11.

The new SOLPS solution had the pumping rate of 340 A, very close to the experimental value. The comparison between the simulated and measured outer target profiles is presented in Figs. 12-13. Large discrepancy in both $n_{e}$ and $T_{e}$

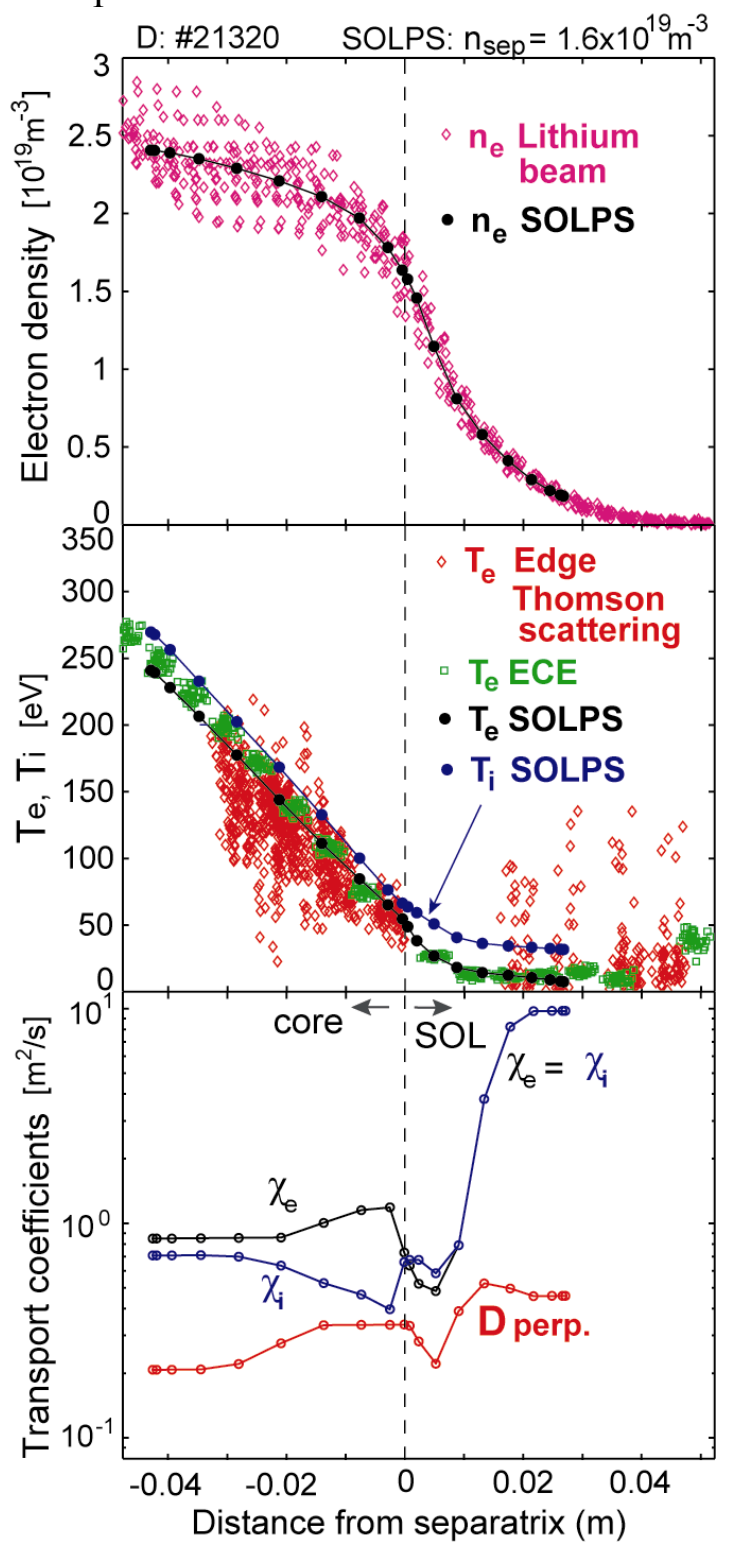

Fig. 11. Experimental and code simulated profiles of $n_{e}, T_{e}$, simulated $T_{i}$ profile, and perpendicular transport coefficients used to obtain the fits, for the case with $n_{\text {sep }}=1.6 \times 10^{19} \mathrm{~m}^{-3}$ (see text for details). 
profiles can be seen, consistent with the previous modelling. Failure of the code to match the peak in target $T_{e}$ profiles is a typical feature of all SOLPS solutions that satisfy main plasma boundary conditions and fit upstream profiles. The case shown in Figs. 11-13 was run with chemical sputtering switched off, leaving only physical sputtering, resulting in an almost pure deuterium plasma. Specifying a non-zero chemical sputtering coefficient would further

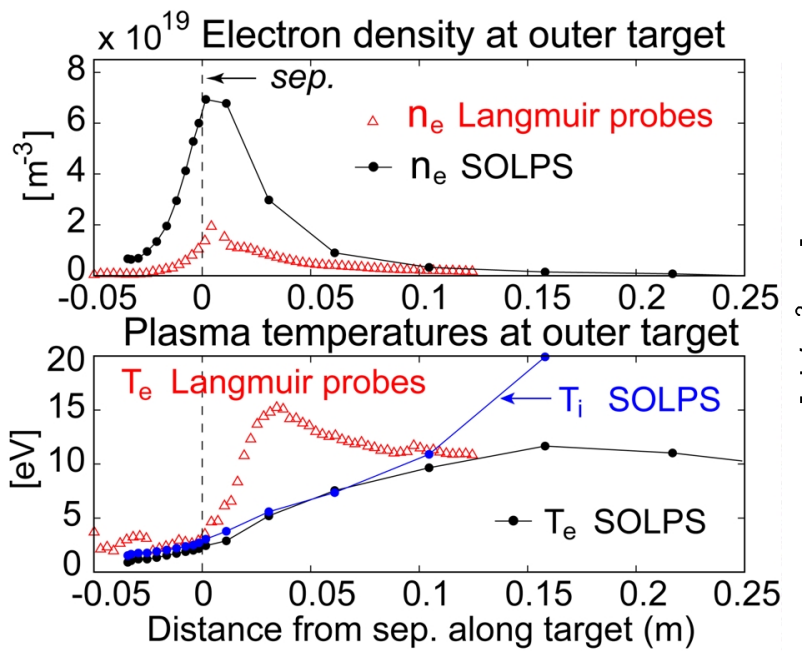

Fig. 12. Measured and simulated outer target profiles of $n_{e}$ and $T_{e}$, simulated $T_{i}$ profile at the outer target for the case with $n_{\text {sep }}=1.6 \times 10^{19} \mathrm{~m}^{-3}$.

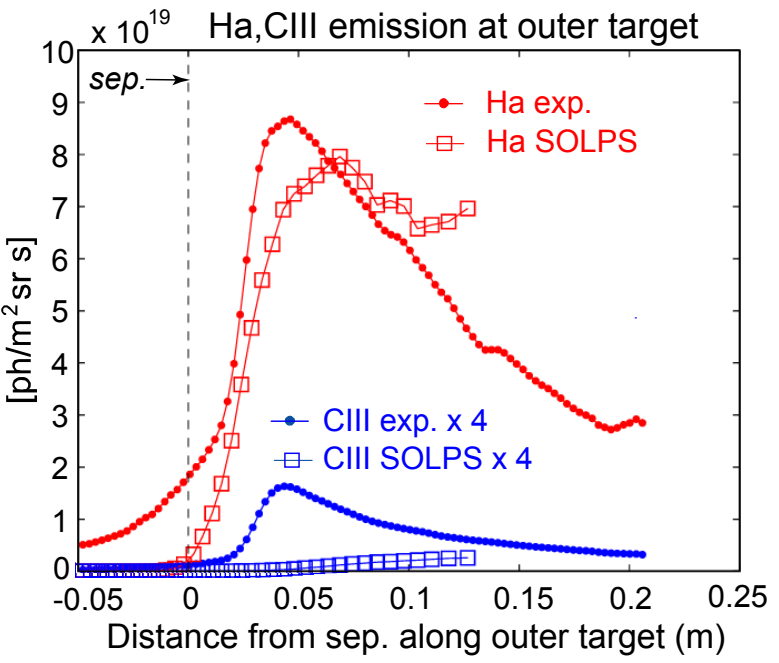

Fig. 13. Measured and simulated $H_{\alpha}$ and CIII emission profiles across the outer target for the case with $n_{\text {sep }}=1.6 \times 10^{19} \mathrm{~m}^{-3}$. The position of divertor spectroscopic chords is indicated in Fig. 1.

reduce the target $T_{e}$ aggravating the discrepancies with the experiment. The simulated $H_{\alpha}$ profile at the outer target matches the experiment very well (see Fig. 13). This, however, must be viewed as fortuitous given that $n_{e}$ and especially $T_{e}$ for Fig. 12 used in Fig. 13 are so different. As in the low density case, the simulated $\mathrm{P}_{\text {rad }}$ agrees with the experimental value, but for the high density case with zero chemical sputtering, edge radiation is almost entirely on deuterium, with negligible contribution from carbon. The simulated CIII radiation shown in Fig. 13 is therefore meaningless and should not be compared with the experimental data. At the inner target, despite a substantially higher $n_{\text {sep }}$, SOLPS simulations again, as in the low density case, did not predict strong detachment. The peak $n_{e}$ at the inner target was $8.4 \times 10^{19} \mathrm{~m}^{-3}$, with $\mathrm{T}_{\mathrm{e}}$ somewhat below $1.5 \mathrm{eV}$ in the vicinity of the strike point.

As one can see, it is impossible to fit both upstream (in the SOL) and downstream (in the divertor/target) profiles in the same SOLPS run, indicating possible problems with the model employed in the present SOLPS package. The shift of $6.5 \mathrm{~mm}$ between upstream $T_{e}$ and $n_{e}$ profiles makes a significant difference to the code solution. This shift, however, is within experimental uncertainty, as stated earlier. It is also within experimental uncertainty of the determination of the separatrix position. The later can be determined by the code using power balance, by the requirement that the code calculated power to the target should match the 
experimental value. The separatrix position determined in this way is affected by the choice of the model for the parallel heat transport used in the code (Braginskii's parallel heat conductivities are presently used). The failure of the code to match the experiment may therefore indicate that this model is not adequate for plasmas in the SOL, for example due to their insufficient collisionality (see e.g. [9] and refs. therein).

One comment should be made regarding the magnetic equilibrium of the simulated plasmas. The chosen position of the separatrix with respect to the outer limiter (the 'outer gap') is the result of a compromise between the desire to have as large outer and inner gaps as possible (since the code treats plasma interaction with the target better than with the wall) and the need to cover the plasma edge by the vertical YAG laser chords that in AUG are located close to the outer limiter (at midplane position). The equilibrium used for the shot $\# 21320$ simulated here has a slightly smaller outer gap than in the previously simulated shot \#18737 (see [8]), with the plasma being very close to the upper part of the RF limiter (see Fig. 1). As a result, a non-negligible amount of power and particle flux was deposited at the wall. For the low density case $\left(\mathrm{n}_{\mathrm{sep}}=1.2 \times 10^{19} \mathrm{~m}^{-3}\right), 34 \%$ of the ion flow and $10.7 \%$ of the power flow through the separatrix reached the wall. For the high density case $\left(\mathrm{n}_{\mathrm{sep}}=1.6 \times 10^{19} \mathrm{~m}^{-3}\right)$ the corresponding figures were 27 and $13.2 \%$. In both cases, however, the particle flow to the wall represented only $3 \%$ of the total recycling flow at the targets. One can therefore assume that the influence of the small gaps in the present configuration on the divertor and target parameters was not significant. This was also confirmed in the experiment, where no significant variation in edge profiles were observed during horizontal sweeps in the vicinity of the gaps of the magnetic configuration described in this paper.

\section{Summary}

SOLPS simulations of the dedicated AUG Ohmic shot at medium plasma density extended an earlier study based on the standard AUG Ohmic shot [8]. The magnetic equilibrium was changed to allow for collection of the divertor spectroscopic array data, covering outer and inner strike points. A diagnostic malfunction however has not allowed to determine absolute positions of measured upstream $n_{e}$ and $T_{e}$ profiles with respect to each other. This introduced a certain degree of freedom in fitting upstream profiles by the SOLPS code, leading to a family of possible code solutions depending on the value of a shift between the $n_{e}$ and $T_{e}$ profiles (the shift of only $6.5 \mathrm{~mm}$ was sufficient to drastically change the outer target $\mathrm{T}_{\mathrm{e}}$ profile). The tendency of the code to underestimate target $T_{e}$ and overestimate $n_{e}$ for solutions that satisfy main boundary conditions (on the input power into the computational domain, particle throughput and radiated power) and match upstream profiles, was confirmed. A solution aimed at matching the measured outer target $T_{e}$ profile required too low separatrix electron density $n_{\text {sep }}$ that was inconsistent with the experimental particle throughput. Such a 
solution required plasma 'leakage' through the inner core boundary necessary to maintain an artificially low $n_{\text {sep }}$ in the presence of the gas puff rate that was consistent with the experiment. The opposite solution, where the emphasis was made on matching global particle balance and experimental neutral pumping speed, predicted too low outer target $\mathrm{T}_{\mathrm{e}}$ and high $n_{e}$, compared with the experiment. The two solutions almost completely cover the range of possible shifts between measured upstream $n_{e}$ and $T_{e}$ profiles. These results call for a wider discussion of missing elements in the present-day 2D edge fluid codes (e.g. inadequate treatment of non-local kinetic effects of parallel plasma transport or the role of plasma fluctuations) within the edge modelling community.

In the present modelling, a more realistic treatment of neutrals in Eirene was carried out, with the introduction of a baffle between sub-divertor and pumping regions that allowed to match the experimentally measured ratio of neutral fluxes between these regions. A substantial variation in the sub-divertor neutral flux caused by varying the baffle width however made only very limited impact on target parameters, especially at the outer target, provided separatrix density $\mathrm{n}_{\text {sep }}$ was unchanged. In a separate test, two methods of fuelling: by external gas puffing, and by plasma fuelling from the core keeping the same $\mathrm{n}_{\mathrm{sep}}$, were compared with each other. For the same transport coefficients, nearly identical $n_{e}$ and $T_{e}$ upstream profiles in the SOL and outer target profiles were obtained. This confirms a key role played by the input power into the computational domain and separatrix density in determining plasma parameters at the target, at least for a given magnetic equilibrium and target/divertor geometry. 


\section{References}

[1] Schneider R., Reiter D., Zehrfeld H.P., Braams B., Baelmans M., et al., J. Nucl. Mater. 196-198 (1992) 810.

[2] Reiter D., J. Nucl. Mater. 196-198 (1992) 80.

[3] Coster D., Bonnin X., Borrass K., et al., in Fusion Energy 2000 (Proc. $18^{\text {th }}$ Int. Conf. Sorrento, 2000) (Vienna: IAEA) CD-ROM file EXP4/20 and http://www.iaea.org/programmes/ripc/physics/fec2000/html/node190.htm

[4] Coster D., Bonnin X., Braams B., Bürbaumer H., Kaveeva E., et al., in Fusion Energy 2002 (Proc. 19 ${ }^{\text {th }}$ Int. Conf. Lyon, 2002) (Vienna: IAEA) CD-ROM file TH/P2-13 and http://www.iaea.org/programmes/ripc/physics/fec2002/html/node433.htm

[5] Kukushkin A S, Pacher H D, Kotov V, Reiter D, Coster D and Pacher G W, Nucl. Fusion 47 (2007) 698.

[6] Reiter D., J. Nucl. Mater. 196-198 (1992) 80.

[7] Chankin A V, Coster D P, Dux R, et al., Plasma Phys. Control. Fusion 48 (2006) 1839.

[8] Chankin A V, Coster D P, Dux R, et al., J. Nucl. Mater. 363-365 (2007) 335.

[9] Chankin A V, Coster D P, Asakura N, et al., Nucl. Fusion 47 (2007) 479.

[10] Chankin A V, Coster D P, Asakura N, et al., Nucl. Fusion 47 (2007) 762.

[11] Wischmeier M, Coster D, Chankin A, et al., 'Simulating divertor detachment of ohmic discharges in ASDEX Upgrade using SOLPS: the role of carbon', $34^{\text {th }}$ EPS Conf. on Plasma Physics and Controlled Fusion (Warsaw, Poland, July 2007) ECA Vol.31F, P1.040 (2007).

[12] Wischmeier M, Bonnin X, Coster D, Chankin A V, Kallenbach A, Müller H W, and the ASDEX Upgrade Team, Contrib. Plasma Phys., 48 (2008) 249.

[13] Neu R, et al., Plasma Phys. Control. Physics 49 (2007) B59-B70.

[14] Kukushkin A S, Pacher H D, Pacher G W, Janeschitz G, Coster D, Loarte A and Reiter D, Nucl. Fusion 43 (2003) 716.

[15] Fischer R, Wolfrum E, Schweinzer J, ASDEX Upgrade Team, 'Probabilistic Lithium beam data analysis', $34^{\text {th }}$ EPS Conf. on Plasma Physics and Controlled Fusion (Warsaw, Poland, July 2007) ECA Vol.31F, P5.077 (2007).

[16] Lisgo S, Börner P, Boswell C, et al., J. Nucl. Mater. 337-339 (2005) 139.

[17] Rohde V, Mertens V, Loarer T and ASDEX Upgrade Team, 'Gas Balance in High Density Discharges at ASDEX Upgrade', $34^{\text {th }}$ ES Conf. on Plasma Physics and Controlled Fusion (Warsaw, Poland, July 2007) ECA Vol.31F, P2.030 (2007). 